\title{
Effects of Tribological Loading History on the Expression of Tribological Function of Regenerated Cartilage
}

\author{
Koji Yamamoto ${ }^{1)}$, Riki Takaya ${ }^{1)}$, Yasushi Tamada $^{2)}$ and Naohide Tomita ${ }^{1)^{*}}$ \\ ${ }^{1)}$ Graduate School of Engineering, Kyoto University \\ Yoshida-Honmachi, Sakyo-ku, Kyoto, Kyoto 606-8501, Japan \\ ${ }^{2)}$ National Institute of Agrobiological Sciences \\ 1-2, Ohwashi, Tsukuba, Ibaraki 305-0851, Japan \\ *Corresponding author: ntomita@iic.kyoto-u.ac.jp
}

( Manuscript received 8 January 2008; accepted 31 March 2008; published 15 April 2008 )

(Presented at JAST Tribology Conference Saga, September 2007 )

\begin{abstract}
In this paper, effects of tribological stimulations for tissue regeneration on the expression of tribological functions were investigated. Chondrocyte-fibroin constructs were cultured by using the stirring chamber, which can apply tribological movement to the surface of the specimens. Five culture conditions were prepared by the combination of static incubation (S) and stirring culture (D) as follows: S-, D-, SS-, DS- and DD-culture group. From the results of friction tests, it was found that the friction durability in DS-culture group was significantly improved, compared to that in the other groups. However, there was not a significant difference in glycosaminoglycan content between DS-culture and DD-culture group. These results would indicate that the evaluation of tribological performances on regenerated cartilage tissues is not always consistent with the synthesis of extracellular matrix, and that the tribological movement in the pre-conditioning period induces the functional expression and the post-conditioning static incubation can reinforce the effect. Long-term tribological movement, however, has a potential to induce detrimental effects on the surface properties of the regenerated tissues. These findings would be useful information for a clinical application of tissue-engineered cartilage and for an elucidation of the mechanism on a formation of tribological performances in cartilage.
\end{abstract}

Keywords: regenerated cartilage, friction durability, start-up friction, stirring chamber, tissue engineering

\section{Introduction}

The structure and the functions of cartilage tissue are constructed and modulated in the process of tissue formation through the interaction with the internal and external environment. Physical environments surrounding articular joint are well known to play important roles in the synthesis of extracellular matrices (ECMs) and in the maturation of mechanical functions. In regenerative medicine for cartilage repair, recent efforts have focused on developing culture systems of cartilaginous tissue in vitro.

In our previous study, it was shown that the initial lubrication properties of cartilage tissues regenerated under a static incubation gradually approximated to those of intact articular cartilage with an increase of culture period, but they exhibited a weak frictional durability owing to an immature surface network of the $\mathrm{ECM}^{1)}$. In consideration of physical environment for articular cartilage, stirring-chamber culture which applies a relative tribological movement for the tissue surface was developed ${ }^{2}$ and it was found that the culture method could inhibit an expression of fibroblast-like cells on the tissue surface. In addition, 2-week cultured tissues using the chamber exhibited good integration and evaluation score in in-vivo transplantation test at 3 months after allografted into a defect of rabbit knee joint, compared to the statically incubated tissues. However, the effect of the tribological movement on the function of in-vitro regenerated tissues has not been clarified yet.

Our motivation in this study is to investigate effects of tribological loading history, including loading time and period, on the expression of lubrication properties of cartilage tissues regenerated using the stirring chamber, and to examine a relation between frictional property and matrix synthesis. 


\section{Experimental methods}

\subsection{Chondrocyte isolation and seeding}

Articular cartilage tissues were aseptically harvested from the proximal humerus, distal femur and proximal tibia of 4-week-old Japanese white rabbits (Oriental Bio Service Co., Ltd., Japan). Chondrocytes were isolated via enzymatic digestion ${ }^{2)}$. After a single-cell suspension was obtained, the cells were cultured on T-flask $\left(75 \mathrm{~cm}^{2}\right)$ for 5 days with Dulbecco's modified Eagle medium (DMEM) (Nacalai Tesque, Inc., Japan) containing 10\% (v/v) FBS and $1 \%(\mathrm{v} / \mathrm{v})$ penicillin-streptomycin (antibiotics). The cells were seeded onto fibroin scaffold ${ }^{1,2)}$ (diameter: 8 $\mathrm{mm}$, thickness: $1 \mathrm{~mm}$ ) using a concentrated cell suspension method at the cell concentration of $5.0 \times 10^{5}$ cells/scaffold. One of the advantages of the fibroin sponge is that tribological performances of regenerated cartilage can be examined easily because the fibroin sponge has enough strength to be installed on the experimental apparatus and well-differentiated cartilage layer is formed on the surface of the scaffold.

\subsection{Cell culture using stirring chamber}

A cell-tissue co-culture magnetic stirring system (stirring chamber) was used to apply a relative tribological movement to the surface of the chondrocyte-fibroin constructs ${ }^{2)}$. Figure 1 shows a schematic drawing of the culture method using the stirring chamber. The chamber consists of a Teflon stirrer with 10-penetrating holes (diameter: $8.7 \mathrm{~mm}$ ) and a stirring drive of power control unit (VARIOMAG, FL, USA). The chondrocyte-fibroin constructs at 3 days after seeding were set into the holes with the surface of cell seeding side contacting to the bottom of a glass Petri dish. Two pieces of stainless mesh (15 mg each) were placed onto the opposite side of each specimen to prevent floating. $16 \mathrm{~mL}$ of culture medium which contains DMEM, 10\% (v/v) FBS, 1\% (v/v) antibiotics and 1\% (v/v) vitamin $\mathrm{C}$ was added into the dish, and the culture medium was changed every 2 days. Stirring frequency was set at $5 \mathrm{rpm}$, and sliding velocity at the center of specimen was $12 \mathrm{~mm} / \mathrm{s}$. A continuous sliding movement ( $24 \mathrm{~h} /$ day) was applied to the specimens.

\subsection{Design of loading history}

Five groups were prepared for investigating the effect of stirring stimulations on the tribological functions of regenerated cartilage. All of chondrocyte-fibroin constructs were statically incubated with 24-well plate for 3 days after seeding ( $A d$ period) to avoid an initial detachment of chondrocytes due to the stirring stimulation. Loading history was designed as shown in Fig. 2. Figure 2 represents the order and the period of each culture method. S-culture group represents that after Ad period, the specimens were additionally incubated with 24-well plate for 4 days, and in D-culture group, the specimens were set into the stirring chamber and dynamically cultured under the tribological movement for 4 days after $A d$ period. SS-, DS- and DD-culture

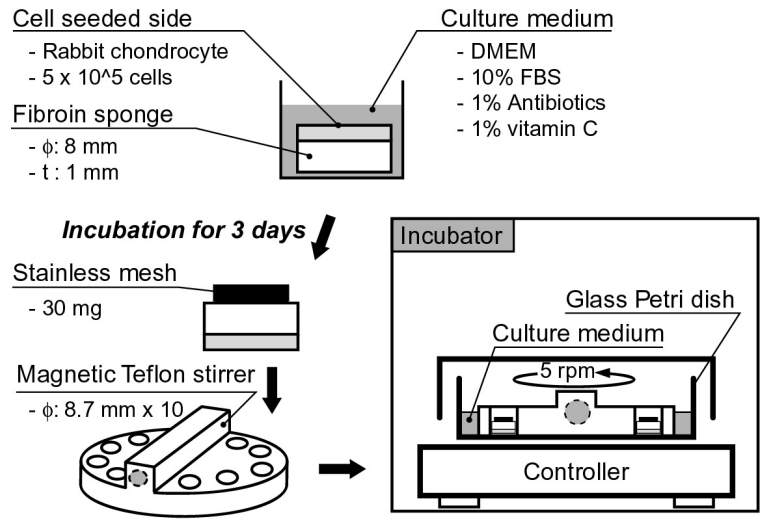

Fig.1 Schematic diagrams of the culture method using the lab-made stirring chamber ${ }^{2)}$

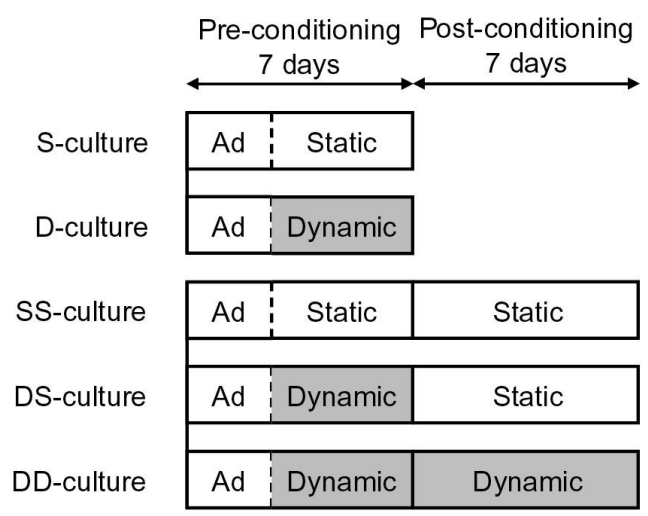

Fig.2 Design of loading history. Static: static incubation with 24-well plate. Dynamic: dynamic culture using the stirring chamber

group means that after pre-conditioning S- or D-culture period, the specimens were additionally cultured for 7 days under post-conditioning static (SS and DS) or dynamic (DD) culture, respectively. S- and SS-culture group means that the specimens were only statically incubated without tribological movement.

\subsection{Measurement of tribological performances}

Friction tests were performed using the regenerated tissues at 7 (S- and D-culture group) and 14 (SS-, DSand DD-culture group) days in culture. The friction force between regenerated cartilage tissue and flat stainless steel (Ra: $0.06 \mu \mathrm{m})$ was measured by using the experimental apparatus shown in Fig. 3. Figure 4 shows the result of kinetic friction coefficient between bovine articular cartilage (diameter: $6 \mathrm{~mm}$, thickness: $1 \mathrm{~mm}$ ) and the stainless steel measured by the apparatus (sliding velocity: 0.008, 0.08, 0.8, $8 \mathrm{~mm} / \mathrm{s}$, normal load: $29.4,294$, $2940 \mathrm{mN}$ ). The friction coefficient clearly showed a positive correlation to the sliding velocity under low normal load, despite the coefficient exhibited significantly high values. These results would suggest that the frictional performances under low normal load 
are associated with some interfacial properties, such as macromolecular interactions and hydration ${ }^{3,4)}$ on the cartilage surface, and at high normal load, they are also affected by tissue deformation and weeping. In consideration of those characteristics and of the stiffness on regenerated cartilage tissue, the experimental conditions of continuous reciprocal sliding and normal load were determined as follows: the sliding velocity was $0.8 \mathrm{~mm} / \mathrm{s}$; the normal load was $29.4 \mathrm{mN}$. All friction tests were performed at room temperature $\left(25^{\circ} \mathrm{C}\right)$ and a saline solution was used as a lubricant. In the experiment, transitions of friction coefficient as a function of duration time, start-up friction and short-term friction were examined. Short-term friction was defined as an average friction during the first 20 seconds after the beginning of a test. Pre-loading time before starting a friction test was set at one minute ${ }^{5)}$.

\subsection{Assay of sulfated GAG content}

The amount of GAG content in regenerated cartilage tissues was assayed using the 1,9-dimethylmethylene blue (DMMB) method. Each chondrocyte-fibroin construct was digested with $1.5 \mathrm{~mL}$ of $24 \mathrm{mg} / \mathrm{mL}$ papain solution prepared with PBS at $60^{\circ} \mathrm{C}$ for 3 hours. The original sample solution was diluted with PBS and DMMB solution was added so that the ratio of the original sample solution to DMMB solution was 1:20, and the absorbance at $525 \mathrm{~nm}$ was measured by using a multi-spectrophotometer (Viento, Dainippon Sumitomo Pharma Co., Ltd., Japan). The amount of GAG in each specimen was calculated using a standard curve calibrated by using a solution of chondroitin sulphate $\mathrm{C}$ sodium salt (Nacalai Tesque, Inc., Japan).

\subsection{Statistical analysis}

Culture experiments were performed in triplicate, and representative results acquired in a single set of culture were shown in the figures because of the same tendency in all culture experiments. The significant difference in GAG content between DS- and DD-culture group was determined by Mann-Whitney U test.

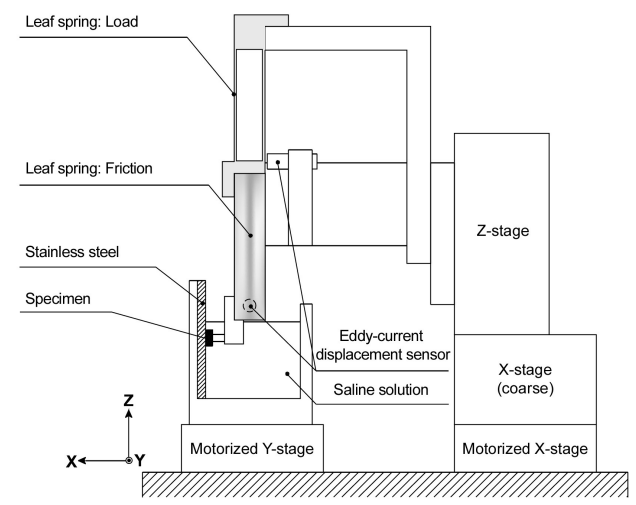

Fig.3 A schematic drawing of the experimental apparatus for friction test

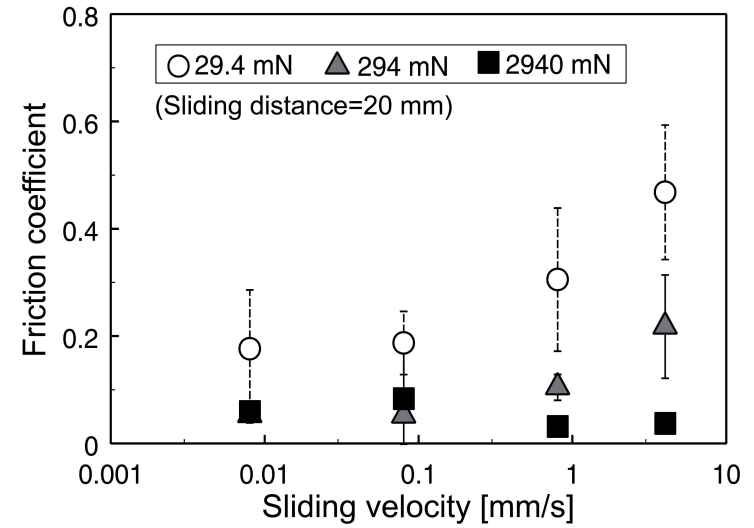

Fig.4 Lubrication properties of bovine articular cartilage for flat stainless steel $(0.06 \mu \mathrm{m} R a)$ in saline solution. Sliding velocity: $0.008,0.08$, $0.8,8 \mathrm{~mm} / \mathrm{s}$ (logarithmic plot), Normal load: 29.4, 294, $2940 \mathrm{mN}$. $(\mathrm{n}=3$, mean \pm SD. $)$

\section{Results}

Transitions of friction coefficient of the regenerated tissues were displayed in Figs. 5(a) and (b). In a comparison of the results between S- and D-culture group (Fig. 5(a)), it was found that the friction coefficient in D-culture group tended to be higher than that in S-culture group. Similarly, DD-culture group showed higher values than SS-culture group (Fig. 5(b)), and the transitions of friction in those groups appear to have the same tendency of a rapid increase to test duration. However, the tissues of DS-culture group showed a much gentle increase in friction, compared to those of the other groups.

The results of start-up friction and short-term friction are shown in Fig. 6. The results of start-up friction indicate that the tissues cultured under tribological movements in the pre-conditioning period (D, DS and $\mathrm{DD})$ tended to be high values, compared to $\mathrm{S}$ - or SS-culture tissues. In DS-culture group and intact bovine articular cartilage (AC), short-term friction exhibited lower values than start-up friction in all specimens.

Figure 7 shows the results of GAG content in DS- and DD-culture group. The data acquired in the first and the second culture experiment was displayed. Although the amount of GAG content showed different values between culture times, there was no difference between DS- and DD-culture group, despite the frictional properties exhibited a significant difference.

\section{Discussion}

The metabolic activity of chondrocytes can be regulated in response to mechanical, electrical, and chemical signals induced by changes in their physical environments. Tissue functions in articular cartilage can be expressed and maintained through the interaction 


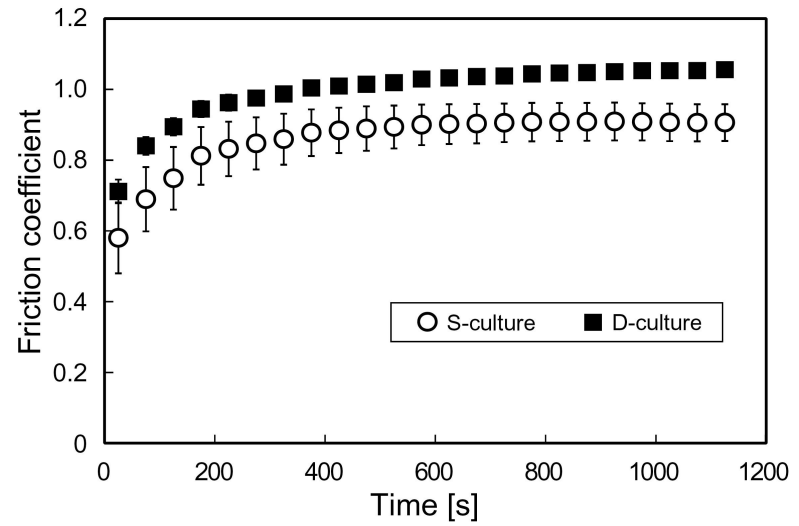

(a) S-culture and D-culture group

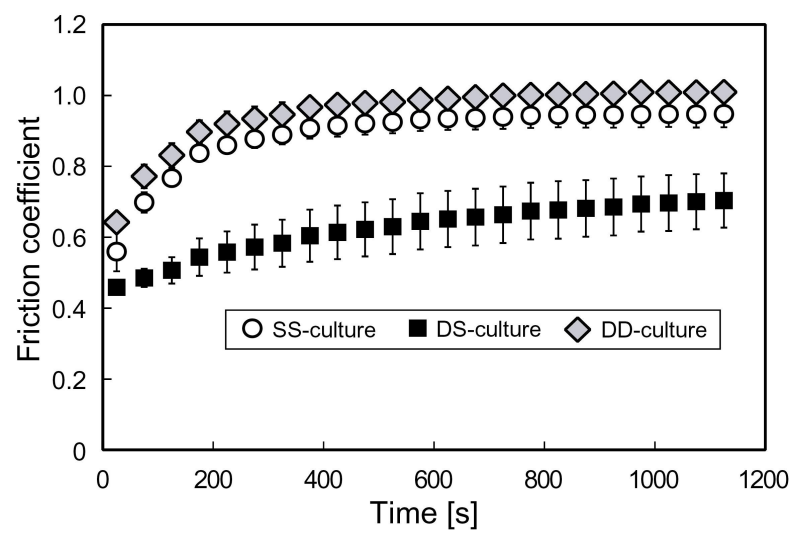

(b) SS-culture, DS-culture and DD-culture group

Fig.5 Time-dependent transitions of friction coefficient of regenerated cartilage. (a) Comparison between S-culture and D-culture group at 7 days after seeding. (b) Comparison between SS-culture, DS-culture and DD-culture group at 14 days after seeding. $(n=3$, mean \pm SD. $)$

among those biological cell characteristics and further, they can be macroscopically influenced by the dynamic structural changes of their ECMs. In this study, effects of tribological loading history for tissue regeneration on the expression of tribological functions were examined.

From the results of the friction test between SS- and DS-culture group, it was suggested that tribological movements in the pre-conditioning period played an important role in the expression of tribological functions, especially friction durability, during the process of tissue regeneration. However, the results in D- and DD-culture group would indicate that the long-term tribological movements in the post-conditioning period negated the pre-conditioning effect for a formation of tribological functions. In addition, when considering the result of GAG content in DS- and DD-culture tissues, long-term tribological movement in this culture might have detrimental effects specifically on the surface properties. Morita et al. exhibited that a weak friction durability of regenerated cartilage tissues probably resulted in an easy exudation of surface water due to an immature matrix

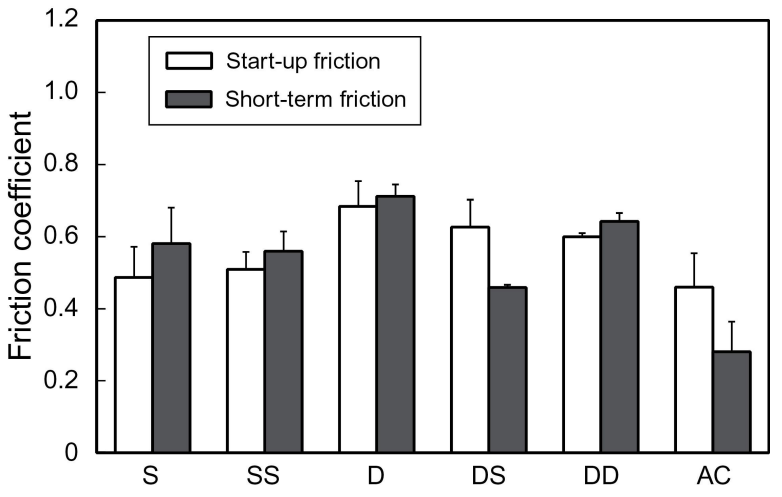

Fig.6 The results of start-up friction and short-term friction of cartilage tissue. AC: intact bovine articular cartilage. $(n=3$, mean + SD.)

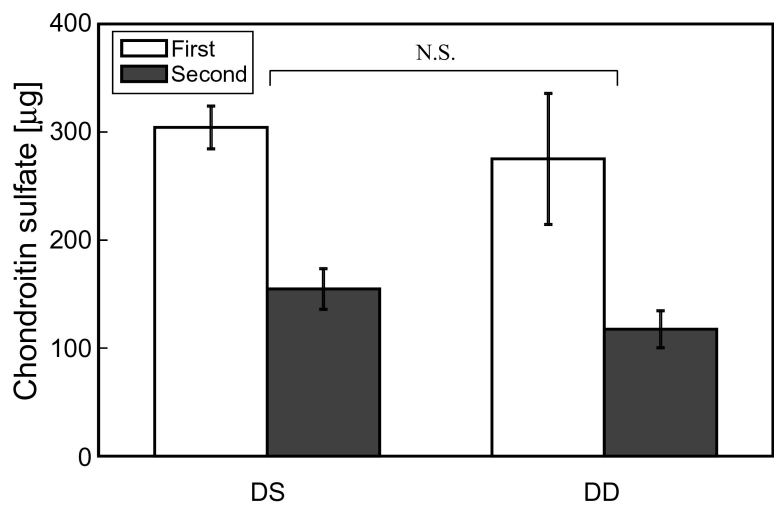

Fig.7 Comparison of the GAG content between DSand DD-culture group. ( $\mathrm{n}=3$ at each experiment, mean \pm SD.)

network ${ }^{1)}$. According to the study investigating the roles of glycoproteins and phospholipids on the frictional properties of articular cartilage, it was suggested that lipids on the surface of articular cartilage have an influence on reducing the friction at low contact pressure with short periods of static loading, and was also demonstrated that removing of surface proteins could cause the increase of start-up friction ${ }^{6)}$. Hence, the improvement of friction durability and the temporal reduction between start-up friction and short-term friction in DS-culture tissues would be ascribed to stimulatory changes in biological and mechanical properties of the tissue surface layer.

It has been reported that PRG4 molecules mediated, at least in part, the lubrication function of the articular cartilage surface layer ${ }^{7-9)}$. In addition, lubricin and superficial zone protein (SZP), which are products of PRG4 gene and surface specific glycoproteins, are known to reduce friction in latex-on-glass ${ }^{9)}$, cartilage-on-glass ${ }^{7,8)}$, and cartilage-on-cartilage. The expression of PRG4 in chondrocytes is also associated 
with the joint motion ${ }^{10)}$. Nugent et al. demonstrated that dynamic shear stimulation could induce PRG4 expression in chondrocytes at a depth of 200-400 $\mu \mathrm{m}$ from the bovine articular surface, and the effect was maintained for 3 days after the load was removed ${ }^{11)}$. In this study, direct shear and/or flow-induced shear stress could be applied to the surface of the chondrocytes and tissues. It could be thought that in DS-culture group, tribological movement in the pre-conditioning period induced the expression of some substances including PRG4 products and the effect was reinforced following static incubation, which involved in the formation of hydrated layer.

Tribological movements are thought to induce functional expressions on tribological performances of cartilage. The results demonstrated in this study indicate an importance of maturation period without external stimuli to more reinforce the functions. Although additional studies are needed to elucidate the molecular details including ECM structure, surface glycoproteins and phospholipids, these findings would be useful information for a clinical application in tissue engineering.

\section{Conclusion}

In this study, effects of tribological stimulations on the expression of tribological performances of regenerated cartilage were examined. It was found that tribological movements in the pre-conditioning period can lead to the effective tribological functions and the post-conditioning incubation can reinforce the effects under in-vitro cartilage regeneration. However, long-term tribological movements probably induced the detrimental effects on the surface properties of regenerated tissues, instead of macroscopic wear of the tissues.

\section{Acknowledgement}

Supported by Knowledge Cluster Initiative Nanotechnology Project and Center of Excellence for Research and Education on Complex Functional Mechanical Systems.

\section{References}

[1] Morita, Y., Tomita, N., Aoki, H., Sonobe, M., Wakitani, S., Tamada, Y., Suguro, T. and Ikeuchi,
K., "Frictional Properties of Regenerated Cartilage in Vitro," J Biomech., 39, 1, 2006, 103-109.

[2] Chueh, S., Tomita, N., Yamamoto, K., Harada, Y., Nakajima, M., Terao, T. and Tamada, Y., "Transplantation of Allogenic Chondrocytes Cultured in Fibroin Sponge and Stirring Chamber to Promote Cartilage Regeneration," Tissue Engineering, 13, 3, 2007, 483-492.

[3] Sasada, T., "Lubrication of Human Joints-Nature of Joint Friction and "Surface Gel Hydration Lubrication",, J. Jpn. Soc. Clinical Biomech., 21, 2000, 17-22 (in Japanese).

[4] Ikeuchi, K., Naka, M. H., Asahi, S. and Fuwa, Y., "Mechanism of Hydration Lubrication in Human Joints,” Proc. Int. Tribol. Conf., Kobe 2005, 2005, 337.

[5] Yamamoto, K., Morita, Y., Kai, M., Ikeuchi, K., Tamashima, Y., Koizumi, T., Tsujiuchi, N. and Tomita, N., "Tribological Performance of Regenerated Cartilage," Jpn. J. Clinical Biomech., 26, 2005, 97-101 (in Japanese).

[6] Pickard, J. E., Fisher, J., Ingham, E. and Egan, J., "Investigation into the Effects of Proteins and Lipids on the Frictional Properties of Articular Cartilage," Biomaterials, 19, 19, 1998, 1807-1812.

[7] Swann, D. A., Hendren, R. B., Radin, E. L., Sotman, S. L. and Duda, E. A., "The Lubricating Activity of Synovial Fluid Glycoproteins," Arthritis Rheum., $24,1,1981,22-30$.

[8] Swann, D. A., Silver, F. H., Slayter, H. S., Stafford, W. and Shore, E., "The Molecular Structure and Lubricating Activity of Lubricin Isolated from Bovine and Human Synovial Fluids," Biochem J., 225, 1, 1985, 195-201.

[9] Jay, G. D., Haberstroh, K. and Cha, C. J., "Comparison of the Boundary-Lubricating Ability of Bovine Synovial Fluid, Lubricin, and Healon," J Biomed Mater Res., 40, 3, 1998, 414-418.

[10] Schumacher, B. L., Hughes, C. E., Kuettner, K. E., Caterson, B. and Aydelotte, M. B., "Immunodetection and Partial cDNA Sequence of the Proteoglycan, Superficial Zone Protein, Synthesized by Cells Lining Synovial Joints," J Orthop Res., 17, 1, 1999, 110-120.

[11] Nugent, G. E., Aneloski, N. M., Schmidt, T. A., Schumacher, B. L., Voegtline, M. S. and Sah, R. L., "Dynamic Shear Stimulation of Bovine Cartilage Biosynthesis of Proteoglycan 4," Arthritis Rheum., 54, 6, 2006, 1888-1896. 